FORMATION Formation emploi

Revue française de sciences sociales

131 | Juillet-Septembre 2015

Le Bac Pro a 30 ans

\title{
L'enseignement dispensé en bac pro est-il professionnel ? L'exemple du baccalauréat Electrotechnique
}

Is Vocational high school diploma really vocational ? One example from the electrotechnical field.

Ist der Unterricht für das Berufsabitur berufsspezifiziert? Beispiel

Elektrotechnik-Abitur

La enseñanza brindada en el bachillerato técnico ¿es técnica? El ejemplo del bachillerato electrotécnico

Josiane Paddeu et Patrick Veneau

\section{OpenEdition}

Journals

Édition électronique

URL : http://journals.openedition.org/formationemploi/4459

DOI : 10.4000/formationemploi.4459

ISSN : 2107-0946

Éditeur

La Documentation française

Édition imprimée

Date de publication : 15 octobre 2015

Pagination : 41-60

ISSN : 0759-6340

Référence électronique

Josiane Paddeu et Patrick Veneau, «L'enseignement dispensé en bac pro est-il professionnel ?

L'exemple du baccalauréat Electrotechnique », Formation emploi [En ligne], 131 | Juillet-Septembre

2015, mis en ligne le 15 octobre 2017, consulté le 30 octobre 2020. URL : http://

journals.openedition.org/formationemploi/4459; DOI : https://doi.org/10.4000/formationemploi.4459

(c) Tous droits réservés 


\title{
L'enseignement dispensé en bac pro est-il professionnel ?
}

\section{L'exemple du baccalauréat Electrotechnique}

\author{
Josiane PADDEU \\ Sociologue au Centre d'études et de recherches sur les qualifications (Céreq) \\ PatRick Veneau \\ Sociologue au Centre d'études et de recherches sur les qualifications (Céreq)
}

Résumé

\begin{abstract}
L'enseignement dispensé en bac pro est-il professionnel ? L'exemple du baccalauréat Electrotechnique

La création du bac pro visait, notamment, à valoriser l'enseignement professionnel au moyen, en particulier, d'une forme d'alternance sous statut scolaire. Cependant, l'étude des pratiques d'évaluation des enseignants mises en œuvre pour le bac pro Electrotechnique (Eleec) révèle plutôt le caractère technologique de l'enseignement dispensé, qui apparaît au travers de la primauté accordée à "l'étude de système » technique lors des épreuves pratiques. Il se manifeste aussi par une mise à distance des aspects les plus concrets du travail dans les épreuves destinées à évaluer les apprentissages en milieu professionnel.
\end{abstract}

Mots clés : enseignement technique-professionnel, baccalauréat professionnel, évaluation, enseignant, pratique professionnelle.

Abstract

Is Vocational high school diploma really vocational? One example from the electrotechnical field

The vocational high school diploma aimed to increase the status of vocational education, particularly with the institution of sandwich courses. However, an investigation of teacher's assessment practices stresses the technological nature of vocational high school teaching. This technological nature appears through the focus teachers put on technical system study during practical examinations. The way teachers depreciate concrete aspects of work while assessing workplace learning outcomes shows this technological nature too.

Keywords: technical \& vocational education, vocational baccalaureate, evaluation, teacher, professional practice.

Journal of Economic Literature: I 21

Traduction : Auteurs. 
La création du bac pro, en 1985, visait à renforcer le caractère professionnel de l'enseignement, en rapprochant l'école de l'entreprise (Formation Emploi, 1995). Pour cela, deux innovations pédagogiques majeures sont introduites. D’une part, aux anciens « horaires, programmes, instructions ", se substituent des référentiels. Ces derniers énumèrent les compétences à acquérir, définies à partir d'une liste de tâches professionnelles caractéristiques de(s) l'emploi(s) visé(s). L'objectif de l'enseignement n'est pas tant alors de dispenser des savoirs que de " faire acquérir » des " compétences ». D’autre part, est instaurée une forme d'alternance sous statut scolaire : les périodes de formation en milieu professionnel (PFMP dans la suite) ${ }^{1}$.

Cette volonté d'accroître le caractère professionnel de l'enseignement s'est réalisée dans le cadre non seulement d'une augmentation de la durée de formation mais d'une hausse de " niveau ", au sens de la nomenclature des niveaux de formation (1969), autrement dit par le biais de la création d'un diplôme de "niveau IV ". Cela permettait de répondre aux diverses demandes de revalorisation de cet enseignement. En effet, la nomenclature des niveaux de formation (1969), élaborée dans le cadre des $I^{e}$ et $V^{e}$ plans, oriente encore les politiques éducatives concernant le système français de formation initiale (Tanguy, 2002).

Dans la hiérarchie unidimensionnelle des savoirs inhérente à cette nomenclature, le « niveau IV » est défini en référence à des savoirs techniques transmis par des formations techniques ("baccalauréat technique ou de technicien et du brevet de technicien »). Les savoirs professionnels qui caractérisent l'enseignement professionnel sont, quant à eux, situés à un niveau inférieur au niveau IV de cette nomenclature. Faut-il en conclure que la création du baccalauréat professionnel a eu pour effet de faire évoluer la hiérarchie des savoirs ? Autrement dit, assiste-t-on à une revalorisation des savoirs professionnels ? Cela interroge sur ce qui, au-delà des injonctions et autres prescriptions nationales (référentiels, alternance sous statut scolaire), est réellement enseigné et sur la place octroyée aux savoirs professionnels dans cet enseignement.

Le qualificatif de "professionnel ", pour caractériser des diplômes ou un type d'enseignement, est longtemps resté flou et imprécis. Il a d'abord fait l'objet d'une acception institutionnelle pour désigner un enseignement qui, justement, ne conduisait pas au baccalauréat.

Outre cet aspect, les nombreux travaux conduits sur les diplômes professionnels ont abordé le terme de professionnel essentiellement sous l'angle privilégié du rapport à l'emploi. Ainsi est qualifié de professionnel le diplôme qui conduit à un emploi ou des emplois.

1. Cette volonté de rapprochement s'est également traduite par un ensemble de mesures institutionnelles. Les CPC (Commissions Professionnelles Consultatives) en charge de l'élaboration des diplômes sont réorganisées dans le sens d'une plus grande implication des représentants du monde professionnel. Des instances nationales de réflexion et de proposition, comme le Haut Comité Education-Economie (1984), sont par exemple créées (Fourcade et alii, 1992). 
Dans ce cadre, " professionnel " trouve son sens par opposition à d'autres termes, comme propédeutique ou bien général/académique.

D'autres travaux, beaucoup plus rares, ont cherché à caractériser l'enseignement professionnel non plus dans son rapport à l'emploi mais au travail. Ils envisagent alors la place accordée, dans cet enseignement, à la pratique ou l'action (Delbos et Jorrion, 1990 ; Tanguy, 1991 ; Barbier, 1996 ; Darré, 1999 ; Cornu, 2001), et encore la plupart ne l'aborde que de manière indirecte ; l'objet premier étant le travail et les savoirs mobilisés au travail.

Le texte présenté ici s'inscrit dans cette seconde approche. Il ne prend en effet pas pour objet la "politique des diplômes » et les rapports de ces derniers à l'emploi, mais l'enseignement professionnel effectivement dispensé et ses liens au travail, entendu comme activité concrète (Bidet et alii, 2006).

Il aborde cet enseignement à partir des pratiques d'évaluation des enseignants ( $c f$. encadré 1), prises dans leur cadre matériel, règlementaire et plus largement institutionnel. Ces pratiques d'évaluation permettent d'identifier assez précisément les apprentissages valorisés par l'institution et les enseignants. Elles constituent donc une voie privilégiée pour déterminer la manière dont est pris en compte le travail dans l'enseignement. Ainsi, notre approche s'inscrit dans une sociologie des curricula qui, rappelons-le, s'intéresse à la sélection, l’organisation, le mode de transmission des savoir et leur évaluation (Forquin, 2008).

La création du baccalauréat professionnel a concerné en premier lieu le champ de la métallurgie. Ainsi, les débats sur la transformation des organisations productives qui accompagnent l'émergence de ce diplôme concernent d'abord les entreprises des secteurs des biens d'équipement. Dans la grille de classification de cette branche sont créés, par l'accord de 1975 et les avenants de 1980 et 1983, plusieurs échelons de technicien d'atelier, qui faisaient office de cible idéale pour les futurs bacheliers professionnels. L'UIMM (Union des Industries Métallurgiques et Minières) devenant alors l'interlocuteur patronal privilégié du ministère de l'Education nationale (Fourcade, 1997), les trois premiers baccalauréats professionnels créés concerneront la métallurgie. Pour ces raisons, et pour contribuer au questionnement sur le caractère professionnel de l'enseignement associé à ce diplôme, s'appuyer sur l'exemple du baccalauréat professionnel " Electrotechnique, Energie, Equipement Communicants $»^{2}$ (Eleec dans la suite) ne paraît pas dénué de fondement, d'autant qu'il représente le flux de formés le plus conséquent des spécialités industrielles ${ }^{3}$.

2. Il est l'un des trois diplômes créés, les deux autres étant les baccalauréats " MSMA » (maintenance) et "productique mécanique".

3. En 2015, avec un effectif de 16514 inscrits au diplôme, cette spécialité représentait 15,2 \% de l'ensemble des inscrits des spécialités industrielles, soit le double des spécialités de maintenance ou d'électronique. 
Nous montrerons que l'enseignement dispensé pour l'obtention du bac pro Eleec, loin d'avoir un caractère professionnel affirmé au sens où nous l'avons envisagé, s'apparente davantage à un enseignement technologique, c'est-à-dire un enseignement centré sur l'étude de systèmes techniques. En d'autres termes, la représentation de ce qu'est une formation de niveau IV a contribué à façonner le contenu du diplôme.

L'analyse de l'évaluation dans les épreuves professionnelles de ce baccalauréat montre, dans une première partie consacrée aux épreuves pratiques, que l'évaluation et par extension la formation s'appuient sur une conception de l'apprentissage qui relèverait, au mieux, de ce que D. Schön (1994) appelle le "modèle de la rationalité technique ». Dans ce modèle, la résolution de problèmes pratiques procède de l'application de démarches et de techniques. Consacrée aux épreuves s'appuyant sur les PFMP, la seconde partie révèle que, pour évaluer, les enseignants semblent "tourner le dos" au travail dans ses dimensions les plus concrètes, pour se centrer davantage sur les savoirs technico-scientifiques.

\section{Encadré 1 : Matériaux d'enquête et démarche de recueil}

Le travail de terrain sur lequel s'appuie cet article concerne les épreuves professionnelles du baccalauréat professionnel Eleec (Electrotechnique Energie Equipements Communicants). II s'agit plus précisément :

- $\quad$ des « épreuves pratiques », réalisées en établissement scolaire ;

des épreuves fondées sur les PFMP (Périodes de formation en milieu professionnel) et en particulier la présentation orale du dossier.

Pour les deux épreuves, les investigations se sont déroulées dans trois académies et concernaient les candidats d'établissements (11) différents (Lycée ou CFA - Centre de formations d'apprentis) et de centres d'examen (7) (candidats de structures non habilitées).

Plusieurs techniques de recueil de données ont été mobilisées :

La technique de l'observation directe : présence au moment de l'épreuve. Les observations réalisées sont plutôt de type analytique (Chapoulie, 2000). Elles se sont centrées sur les repères (Figari et Tourmen, 2006) dont se servent les enseignants pour évaluer, soit 36 observations concernant près de 100 candidats, complétées par l'observation de 16 TP, et 18 situations (jurys) d'épreuve orale pour 120 candidats environ ;

- Des entretiens réalisés à l'issue des épreuves pratiques (33 entretiens), avec les évaluateurs, ou « entretiens post-épreuve ». Au cours de ceux-ci, nous avons invité les évaluateurs à renseigner, en notre présence, les grilles d'évaluation et à noter, s'ils le pouvaient.

- Des entretiens semi-directifs plus classiques (34 entretiens au total), avec les Inspecteurs territoriaux responsables du diplôme, des chefs de travaux ou responsables pédagogiques de CFA et les enseignants ou formateurs s'agissant des épreuves qui se déroulent dans le cadre du CCF (Contrôle en Cours de Formation).

Toutes les situations d'épreuve ont donné lieu à des enregistrements ou bien à des prises de note, qui ont constitué le matériau de base traité dans ce texte, essentiellement par analyse de contenu. 


\section{Encadré 2 : Un diplôme surtout spécifié par ses épreuves}

Le bac pro Eleec (Electrotechnique Energie Equipements Communicants) succède au baccalauréat « Equipements et Installations Electriques » (EIE) (1985), dont les objectifs en termes d'emploi ne contenaient aucune référence à un quelconque métier ou catégorie socioprofessionnelle. Le référentiel du baccalauréat Eleec (2003) a peu modifié cet aspect. Les objectifs professionnels de ce diplôme sont demeurés relativement indéterminés, avec une définition du « métier » par une discipline : l'électricité. La liste de fonctions restreint peu le domaine d'activité de ce bac, qui s'étend de la réalisation à l'étude, en incluant la maintenance.

En revanche, le contenu de ce diplôme est davantage précisé par les épreuves. De ce point de vue, l'évolution observée a consisté à faire disparaître l'épreuve de "réalisation », qui existait dans le référentiel de 1990. Il y avait alors une épreuve (coefficient 5) intitulée : « évaluation de la pratique professionnelle dans l'établissement de formation », dont l'objectif consistait, entre autres, à « implanter et raccorder des composants ou constituants électriques dans un ensemble ». En 2003, elle est remplacée par trois épreuves : « mise en service », " maintenance », "réglage, paramétrage, contrôle, modifications liées au champ d'application », dont la somme des coefficients est égale au coefficient de l'épreuve « technologique » (coefficient 5).

De ce point de vue, le baccalauréat se singularise très nettement du BEP (brevet d'études professionnelles) en tant que diplôme. Ce dernier comportait en effet une épreuve technologique dont le coefficient 4 représentait environ un tiers seulement de celui affecté à l'épreuve de réalisation (coefficient 11). Cette différence marque l'écart « de niveau » entre le BEP et le baccalauréat professionnel.

Enfin, les différentes évaluations relatives aux PFMP (périodes de formation en milieu professionnel) totalisent un coefficient égal à 3.

\section{L'épreuve de maintenance : une épreuve pratique ou technologique?}

L'observation du déroulement des épreuves pratiques met clairement en évidence l'activité de contournement du référentiel à laquelle se livrent les enseignants. Bien qu'important, cet aspect ne sera pas abordé ici. Les pratiques enseignantes seront envisagées en tant que telles et non dans leur rapport aux prescriptions nationales. En conséquence, les enseignants évaluent ce à quoi ils forment. Cela autorise, comme nous le ferons parfois, des raccourcis entre pratiques d'évaluation et pratiques de formation. Cet aspect fait l'objet d'un autre texte qui envisage notamment le rapport des enseignants aux référentiels et aux grilles nationales d'évaluation (Paddeu et Veneau, 2016) ${ }^{4}$. En outre, si les trois épreuves dites " pratiques » de ce diplôme ont fait l'objet d'investigations, seule l'épreuve de maintenance sera évoquée dans cette partie. Toutefois, ce qui sera avancé au sujet de cette dernière pourrait s'appliquer aux deux autres, comme nous le signalerons à certains moments.

4. Paddeu J., Veneau P. (soummission d'article 2015), «Le travail d'évaluation des enseignants à l'épreuve des politiques éducatives de réussite et de performance et de leurs instruments ". 
Nous montrerons d'abord la place qu'occupent les démarches méthodiques et rationnelles dans le travail demandé aux candidats. Elles sont favorisées et rendues possibles par les caractéristiques des situations d'épreuve (point suivant). En accordant une place conséquente à l'étude de systèmes, l'épreuve de maintenance - comme l'ensemble des épreuves dites "pratiques "- apparait comme une bonne préparation à celle, nationale et écrite, dite « étude d'ouvrage » et dotée d'un coefficient important.

\subsection{Dépanner, une question de méthode}

La finalité de cette épreuve de maintenance est simple. Les enseignants ${ }^{5}$ provoquent une panne sur le circuit électrique d'une installation sur laquelle les élèves sont déjà intervenus ${ }^{6} ;$ ces derniers ont en général trois heures pour la remettre en état de marche. Les référents ou repères des enseignants pour juger et évaluer la prestation des candidats sont principalement relatifs à la " recherche de panne ", pour reprendre leur expression. Dépanner, soit, mais avec méthode. Tel est le credo de l'ensemble des enseignants, sans cesse rappelé aux candidats, parfois même de manière solennelle en début d'épreuve.

"L'important, à mon sens, c'est quills aient la bonne démarche» (formateur de CFA depuis 2003, 43 ans, BTS - brevet de technicien supérieur -, 9 ans d'expérience professionnelle en entreprise)

Ou bien, de manière plus marquée.

" Donc, finalement, qu'ils trouvent la panne ou pas, pour nous, ce n'est pas important. C'est vraiment la recherche de panne » (enseignant LP et GRETA ${ }^{7}, 38$ ans, BTS et licence, admis $\mathrm{CAPET}^{8}$ et $\mathrm{PLP}^{9}$, un an de maintenance industrielle).

5. Par rapport aux travaux de L. Tanguy (1991), on assiste à une homogénéisation du corps enseignant. Les " anciens OP » sont partis. Ils sont actuellement remplacés par une nouvelle population proche des enseignants " diplômés du technique supérieur court ». Toutefois, ils sont titulaires d'un diplôme de deuxième cycle universitaire. Ils ont aussi tenté de passer le CAPET et l'ont parfois obtenu. Cette nouvelle population est ainsi à la fois proche des "diplômés du technique supérieur court ", car ils ont eux aussi obtenu un BTS ou un DUT (diplôme universitaire de technologie), et ils se rapprochent, par leur diplôme et leur parcours, des enseignants des lycées généraux et technologiques : leur expérience du travail en industrie est réduite. On peut donc parler, comme nous le ferons en conclusion, d'une " généralisation " des enseignants de lycées professionnels au sens où les nouvelles générations s'apparentent de plus en plus aux enseignants des lycées généraux (et technologiques).

6. Au moins pour les candidats qui passent cette épreuve dans le cadre du CCF. Ce qui ne signifie pas, comme nous allons le voir, qu'ils « connaissent » bien le système sur lequel ils vont intervenir. C'est d'ailleurs le reproche que leur adressent en permanence les enseignants en cours d'épreuve, tout en les aidant par leurs nombreuses interventions.

7. GRoupement d'ETAblissements. Un GRETA est un groupement d'établissements publics locaux d'enseignement (EPLE) qui fédèrent leurs ressources humaines et matérielles pour organiser des actions de formation continue pour adultes.

8. Concours au «Certificat d'Aptitude au Professorat de l'Enseignement Technique ».

9. Concours (interne ou externe) de Professeur de Lycée Professionnel. 
L'importance de la "recherche de panne " apparaît dans les barèmes que construisent les enseignants. Sur vingt points, ils lui attribuent parfois jusqu’à dix-sept points ; trois restant pour la « remise en état du système» et "intervenir en sécurité ». Mais le plus souvent, sa valeur dans les barèmes oscille entre treize et quinze points. L'objectif "remettre l'ouvrage en état de fonctionnement ", identifié dans le référentiel, devient secondaire. Et on peut se demander si la recherche de panne ne constitue pas une sorte de "situation-problème " - une tâche pour apprendre -, propice à l'étude de systèmes et à la mobilisation de démarches et de savoirs.

La méthode attendue de recherche de panne comprend trois étapes. Et les enseignants veillent à ce que le travail des élèves soit conforme à ce cheminement, en faisant parfois avec eux des "bilans d'étape ".

La première étape, désignée sous le terme de " constat " par les enseignants, consiste à identifier la partie du système qui n'est plus en état de marche. Elle fait appel à l'observation de ce qui ne fonctionne plus mais aussi de ce qui est encore en état de marche. Mais elle suppose surtout que soit conduite une étude technique du système à dépanner. Cette dernière doit être effectuée à partir du " dossier technique " (dossier papier) de l'installation, lequel comprend un descriptif précis de l'installation (son cycle notamment) mais aussi les schémas électriques. Quand ils éprouvent des difficultés, ce qui est courant, les élèves sont systématiquement invités à se reporter à ce dossier qui est censé comporter tout ce dont ils ont besoin pour faire leur recherche de panne. Cette première étape de constat et d'étude de système pour identifier la zone en défaut est, de loin, la plus longue et la plus délicate pour les élèves. Elle conditionne la suite de l'épreuve.

L'étude technique de systèmes n'est pas propre à cette épreuve. Elle est aussi attendue pour les deux autres épreuves pratiques, celle de mise en service et celle de paramétrage, lesquelles lui accordent une place tout aussi importante. On peut dès lors se demander s'il n'existe pas qu'une seule et même épreuve "pratique " qui consisterait en une étude de système . "Dépanner ", " mettre en service " ou bien encore " modifier un ouvrage " ne constitueraient en somme que des prétextes et non des finalités. La place qu'occupe cette étude dans les épreuves pratiques et dans l'épreuve écrite nationale ( $c f$. infra) pousserait à qualifier l'enseignement dispensé de technologique au sens ou Y. Deforge (1991) définit ce terme, à savoir un enseignement centré sur une "étude de systèmes (et des relations entre systèmes)" (p. 405 $)^{10}$.

Elaborée dans les années 60 (Canonge et Ducel, 1969), l'étude technique de systèmes est à la fois forme et substance. Autrement dit, elle est aussi une démarche, une manière d'appréhender les installations techniques complexes mais aussi un moyen pour trans-

10. Pour approfondir cette réflexion sur les notions de technique et de technologique, voir Deforge, 1989. 
mettre les savoirs techniques ${ }^{11}$. Enseignée en lycée technologique (Pelpel et Troger, 1993) et dans l'enseignement supérieur court (STS) (Eckert et Veneau, 2000), elle s'est aussi diffusée dans l'enseignement professionnel (Tanguy, 1991), au moins pour certaines spécialités industrielles (mécanique, maintenance, électronique...) dont celle considérée ici. La plasticité de cette "étude ", son caractère adaptable et transposable à quasiment tous les systèmes techniques, son apparente rationalité, constituent autant d'éléments en faveur de sa diffusion. Elle donne l'illusion de pouvoir s'affranchir des conditions concrètes de réalisation d'une activité professionnelle.

L'étude de systèmes est censée permettre l'identification de la zone électrique en défaut. C'est dans cette première étape que les difficultés rencontrées par les candidats sont les plus vives. Ils tentent de les contourner en élargissant le " périmètre » de la zone électrique qu'ils supposent être responsable de l'arrêt. Par cette tactique, ils tentent de pallier le caractère bien souvent approximatif de leurs constat et analyse. Ce faisant, ils amplifient les difficultés de la deuxième étape.

La deuxième étape consiste à formuler un certain nombre d'hypothèses susceptibles d'expliquer l'arrêt ; le type de panne provoqué par les enseignants est suffisamment large pour en admettre plusieurs. Ce deuxième temps permet aux enseignants de se faire une idée assez précise de la qualité du travail réalisé dans la phase précédente. Leétude de systèmes a-t-elle été bien conduite ? Les candidats savent-ils lire et interpréter des schémas électriques ${ }^{12}$ ? Connaissent-ils bien les symboles électriques ? Accessoirement, savent-ils raisonner et être logiques ? La pertinence des hypothèses, leur caractère vraisemblable ou farfelu, leur nombre... constituent autant de critères d'évaluation. La plus ou moins grande qualité des hypothèses formulées contribue ainsi fortement à déterminer l'intervalle dans lequel se situera la note définitive, et pour beaucoup d'enseignants, l'épreuve pourrait s'arrêter là.

Dans la continuité, la troisième étape, celle que les élèves appréhendent le plus aisément, consiste à vérifier ces hypothèses. C'est-à-dire non seulement prendre des mesures de tension... mais leur donner du sens, les interpréter.

Dans cette épreuve, dépanner est donc principalement envisagé comme une question de méthode, voire de procédure ; ce qu'admettent assez facilement les enseignants, même s'ils mettent en avant la question du temps dont ils disposent et la singularité de l'école :

"Pour nous, l'important, c'est la méthodologie, parce qu'on est des formateurs, on n'est pas sur l'efficacité, ce n'est pas mon objectif. Voilà, je n'ai pas ce problème. Moi l'élève, il faut

11. Plus précisément, l'étude de systèmes techniques consiste en une approche globale puis descendante des systèmes. Elle prône en premier lieu une analyse des fonctions et sous-fonctions du système (analyse fonctionnelle) puis des structures techniques associées à ces fonctions (analyse structurelle), en descendant jusqu’aux constituants les plus élémentaires.

12. Aspect essentiel pour certains enseignants qui n’hésitent pas à avancer que « dépanner, c’est savoir lire un schéma». 
calmement qu'il essaie d'analyser progressivement, même s'il va lentement. Exactement. Il doit mettre en place une procédure qu'on lui a apprise et essayer de la développer. C'est tout. C'est déjà très bien, sachant que ce n'est pas en un trimestre que je vais rendre quelqu'un performant dans la maintenance. C'est une spécialité, la maintenance. » (enseignant de LP, 46 ans, DU - diplôme universitaire de technologie -, enseignement privé, PLP).

On pourrait évoquer ici à nouveau la prégnance du " modèle de la rationalité technique » et la manière dont ce dernier considère "l'agir professionnel " comme une application de savoirs plus généraux. Les travaux de L. Tanguy (1991) montrent que les enseignants diplômés du technique supérieur court sont portés également à envisager "la pratique comme une application [de savoirs techniques] ne présentant pas de difficulté particulière" (p. 141). Nous serions tentés de radicaliser ces propos. Dans cette épreuve pourtant qualifiée de " pratique ", la pratique entendue comme activité visant à résoudre un problème singulier, concret (Barbier, 1996), est minimisée. Elle se dissout dans les démarches à mobiliser. La sous-valorisation de la pratique ne relève pas uniquement d'un modèle pédagogique partagé par les enseignants ; elle tient également aux caractéristiques de la situation d'épreuve. Cette dernière s'impose en grande partie aux enseignants, au travers de préconisations et dotations d'équipements. C'est sur cet aspect que nous insisterons dans la suite.

\subsection{Des démarches facilitées par la situation artificielle de l'épreuve}

Le type d'évaluation pratiqué par les enseignants est à la fois favorisé et permis par la situation $^{13}$ de l'épreuve. Cette dernière opère une très forte simplification des situations standards de dépannage en entreprise. A travers celle-ci se renforce la forme scolaire de cette épreuve. Ainsi, les systèmes sur lesquels interviennent les candidats sont peu complexes. La partie opérative est rudimentaire et par conséquent la taille des armoires électriques réduite. Il s'agit le plus souvent de systèmes didactiques qui rendent possible, aux dires de certains enseignants, un dépannage par tâtonnements vivement proscrit par ces derniers.

Les caractéristiques de ces matériels limitent la variété des pannes envisageables. Ce sont donc toujours un peu les mêmes pannes qui reviennent. D'autant plus que les enseignants privilégient celles qui se prêtent le plus au déploiement de la démarche d'intervention. Elles vont donc porter en priorité sur le circuit de commande - et moins fréquemment sur celui de puissance - qui, relativement aux ouvrages, autorise la formulation d'un plus grand nombre d'hypothèses et ouvre l'espace du raisonnement qu'ils attendent. Les dysfonctionnements de capteur, fréquents dans certains contextes de production, ainsi que les courts-circuits, sont peu utilisés ; quant aux pannes intermittentes, elles ne sont pas envisageables. La mise en défaut de contacteurs, de fusibles, de relais, etc., demeure la panne provoquée la plus répandue. Elle satisfait à ce qui est recherché par les enseignants,

13. Nous reprenons ici le terme de situation dans l'acception élargie qu'en fait L. Quéré (1997) et dans laquelle les objets sont partie prenante de l'action (de dépanner par exemple). 
tout en étant facile à provoquer. Il n'en demeure pas moins que ce type de défaillance est le plus à même d'être anticipé et évité par une maintenance préventive. Ainsi, les pannes auxquelles sont confrontés les candidats sont rares en contexte de production (Formation Emploi, 1995).

Dans les entretiens, les enseignants ont parfois opéré une analogie entre le travail de maintenance et celui d'enquête (de police). Cependant, "l'enquête » que les candidats doivent réaliser comporte rarement des "témoins". En effet, l'épreuve démarre le plus souvent avec le système mis en défaut, les élèves ne savent donc rien du "comment " cela est arrivé. Quelques enseignants construisent néanmoins, en début d'épreuve, un mini scénario. Ils endossent le rôle "d'opérateur de fabrication " qui rend compte de l'arrêt au "technicien ", joué par le candidat. Mais cette mise en scène est peu réaliste, tout comme les pannes. Certains enseignants ont pourtant souligné l'importance de ce moment :

"J'arrive, je consulte le client. En fonction de ce que me dit le client, ça va m'orienter. Je vais essayer de morienter par rapport à ce que me dit le client. Ensuite, après, je vais établir des hypothèses. Quand j'ai établi ces hypothèses-là, j'ai des appareils de mesure... Dans telle hypothèse, je dois prendre tel appareil de mesure pour pouvoir vérifier. Même ce contact là avec le client, je pense qu'il est obligatoire. Il est obligatoire parce que c'est lui qui l'utilise le plus. " (enseignant de LP, 41 ans, BTS et maîtrise, admissible au CAPET, admis PLP, deux ans de travail temporaire en maintenance ${ }^{14}$ )

Privés le plus souvent de " témoins ", les candidats ne disposent pas non plus d'informations sur le système en cours de fonctionnement : quelles sont les zones de fragilité du système, quelles pannes surviennent le plus souvent ? L'absence de tels indices contribue à conférer à l'étude technique et formelle de "l'ouvrage " une place déterminante et à orienter ainsi le travail effectué par les candidats au cours de l'épreuve ${ }^{15}$.

Le rapport au temps constitue le dernier élément qui contribue à rendre abstraite la situation de cette épreuve. Dans nos observations, le temps mis pour dépanner n’est jamais pris en compte dans l'évaluation. Bien au contraire, les candidats sont invités à utiliser tout le temps dont ils disposent pour "peaufiner le compte-rendu de leur intervention, corriger les fautes d'orthographe...». Autrement dit, jamais l'épreuve n'est située dans un horizon d' "efficacité ». Si on peut légitimement supposer qu'au cours de leur

14. On relèvera que lorsqu'ils se remémorent les situations de dépannage qu'ils ont vécues, les enseignants n'évoquent plus l'analyse du système. Un autre enseignant ayant lui aussi travaillé dans le domaine de la maintenance nous racontera comment la prise de mesures peut intervenir très rapidement - ce qu'interdisent les enseignants lors de l'épreuve - pour vérifier qu'il ne s'agit pas d'une situation déjà rencontrée.

15. Une autre caractéristique spécifique des situations d'épreuve observées est relative au fait que jamais la recherche de panne ne se fait en recourant à un système de supervision relié à (aux) l'automate(s) programmable(s) de l'installation. De tels dispositifs aident bien évidemment à la localisation de l'arrêt dans le cycle. 
formation les élèves acquièrent un certain nombre de savoirs à caractère technique et technologique, on peut en revanche se demander quels "savoirs pratiques ", quels "savoirs d'action » ou bien encore quels «savoir-faire » ils peuvent construire dans la préparation à ces épreuves pratiques.

\subsection{Quand la « pratique » est au service de l'épreuve technologique}

Les pratiques d'évaluation observées doivent aussi être replacées dans une économie plus globale des épreuves de ce diplôme et des coefficients qui leur sont affectés. L'importance de l'étude du dossier technique relatif aux installations, pour les trois épreuves pratiques, est à rapporter au contenu de l'épreuve qualifiée de "technologique " et dite " étude d'ouvrage ", épreuve écrite, nationale, présente dans l'ensemble des spécialités industrielles. Cette dernière constitue une des balises les plus fiables de ce qu'il convient d'enseigner (Tanguy, 1991 ; Eckert et Veneau, 2000). Elle contribue en outre de manière décisive - sur le plan pédagogique - à la fixation du «niveau » du diplôme. Signe de son importance, elle est affectée d'un coefficient 5, soit autant que la somme des coefficients des trois épreuves pratiques de ce diplôme ( $c f$. encadré 2). A cet égard, précisons que ce diplôme n’a rien de spécifique, puisque ce rapport est quasi identique dans les autres principaux baccalauréats industriels.

Constituée à partir d'un "dossier sujet " contenant les questions que devront traiter les candidats, l'épreuve d'étude d'ouvrage s'appuie aussi sur un "dossier technique et ressources ». Ce dernier contient notamment le descriptif de l'installation - y compris les schémas électriques - que les élèves doivent étudier en fonction d'une problématique. L'analyse de ce " dossier technique/ressource " est bien évidemment nécessaire pour répondre aux questions posées dans le « dossier sujet ». Chaque épreuve pratique peut ainsi être vue comme un entrânement, une préparation à cette " étude d'ouvrage " où les élèves éprouvent de grandes difficultés. Aux dires des inspecteurs, la moyenne nationale des notes, avant la réforme de 2009, oscillait, selon les années, entre 8 et 10 (sur 20); cette moyenne a bien évidemment chuté lors de la première session du baccalauréat professionnel préparé en 3 ans. Elle a obligé le ministère de l'Education nationale à octroyer deux points supplémentaires à l'ensemble des candidats afin de maintenir un taux de réussite au diplôme "acceptable ", mais néanmoins en forte diminution par rapport aux années antérieures.

Ainsi, les enseignants rappellent aux élèves que le dépannage doit se faire à partir d'une étude du "dossier technique " du système à laquelle ils seront confrontés dans l'épreuve nationale. Pour les enseignants, la contrainte de faire réussir leurs élèves ${ }^{16}$ au diplôme imprègne aussi le déroulement des épreuves. La réduction de la durée de

16. Nous faisons allusion à l'objectif des « $80 \%$ d'une classe d'âge au niveau du baccalauréat ", concomitant à la création du baccalauréat professionnel. Cet objectif pèse d'autant plus qu'il est localement réinterprété par les inspecteurs, l'administration et les enseignants comme un taux de réussite au baccalauréat à atteindre. 
formation avec la réforme dite "rénovation de la voie professionnelle ", le maintien de l'objectif quantifié (les $80 \%$ ), la transformation des publics survenue à l'occasion de la mise en œuvre de cette réforme sont de nature à renforcer les proximités entre l'épreuve nationale et les différentes épreuves pratiques, et la subordination de ces dernières à la première. Ce qui n'est pas de nature à renforcer leur caractère "pratique ".

\section{Malgré les PFMP, une mise à distance du travail au profit des savoirs techniques}

La création du baccalauréat professionnel a introduit une forme d' "alternance sous statut scolaire ", à travers l'instauration des PFMP. (Périodes de formation en milieu professionnel). Dans le cadre de l'épreuve dite "relative aux situations de travail spécifiées et réalisées en milieu professionnel ${ }^{17}$, les situations dans lesquelles sont placés les candidats n'ont donc pas a priori le caractère artificiel des épreuves pratiques. Est-ce donc cette forme d'alternance, plus que toute autre situation d'apprentissage, qui confère à l'enseignement dispensé dans le cadre du bac pro son caractère " professionnel " ? Le déroulement de ces périodes ainsi que leurs divers modes d'évaluation montrent, s'agissant du bac Pro Eleec, que ce n'est pas tout à fait le cas.

Les PFMP sont d'abord évaluées en tant que telles, par les enseignants mais aussi par les tuteurs des candidats en "milieu professionnel». Le candidat doit ensuite produire, à partir des activités qu'il a réalisées au cours des PFMP, un " dossier de synthèse ". Celui-ci doit contenir quatre comptes rendus de situations de travail rencontrées en entreprise, dont l'une fera l'objet d'une "étude de cas " : l'identification et le traitement d'un problème technique en lien avec les activités réalisées. Enfin, le candidat devra présenter oralement ce dossier (en insistant sur l'étude de cas) devant un jury, composé d'un ou plusieurs enseignants (éventuellement un professionnel), avec lequel il s'entretiendra. Cette partie portera surtout sur cette dernière sous-épreuve, même si les deux autres sous-épreuves seront évoquées à l'occasion. Elles constituent en effet le matériau disponible pour l'évaluation dans le cadre de cette épreuve orale.

\subsection{Des apprentissages en milieu professionnel difficiles à évaluer}

Le poids accordé aux PFMP dans la formation conduisant au baccalauréat professionnel est tout relatif : 22 semaines sur l'ensemble des trois années de formation. Au cours de ces périodes de formation, les élèves sont censés réaliser, en situation réelle de travail, les tâches qui sont décrites et énumérées dans le référentiel de diplôme. Cependant, la

17. Nous détaillerons plus loin le contenu de cette épreuve et des sous-épreuves qui la composent, dont une en particulier, l'épreuve orale, constitue l'objet de cette partie. 
recherche d'entreprises d'accueil offrant les activités requises n'est pas aisée ${ }^{18}$ (Agulhon, 2000). Les activités réalisées durant les PFMP consistent plutôt à contribuer à des travaux de réalisation ou d'installation électrique, principalement dans l'habitat ou le tertiaire (bureaux, locaux commerciaux, hôpitaux...) ${ }^{19}$. Ainsi, les élèves y effectuent souvent du "tirage de câbles ", du raccordement de prises électriques ou de luminaires, beaucoup moins souvent d'installation de tableaux électriques ${ }^{20}$. En définitive, et de ce point de vue, il n'existe pas de différence fondamentale entre les activités réalisées par les élèves de CAP et ceux de baccalauréat professionnel ${ }^{21}$.

En fait, les activités effectuées par les «stagiaires » de baccalauréat professionnel, le plus souvent de réalisation, ne constituent qu'une infime partie des activités listées dans le référentiel. Elles ne sont d'ailleurs pas les plus valorisées dans l'évaluation de l'épreuve relative aux " situations de travail spécifiées et réalisées en milieu professionnel ». Ce décalage avec le référentiel n'a rien de surprenant ; il a souvent été mis en évidence par les sociologues (Dossier Formation Emploi, 1995 ; Quenson, 2009).

Un autre décalage est perceptible au travers des évaluations conduites dans le cadre des épreuves évoquées ici. Il s'agit de la différence qu'il peut y avoir entre ce à quoi les élèves sont formés à l'école ${ }^{22}$ (il ne s'agit pas uniquement des contenus des référentiels) et ce qu'ils font et apprennent en situation professionnelle (par exemple en alternance) (Veyrac et Asloum, 2009) ${ }^{23}$. Ce décalage interroge donc le caractère véritablement alterné de cette formation. Tous, élèves, enseignants et tuteurs semblent en avoir pris conscience. Du point de vue des employeurs, ces PFMP sont en effet souvent assimilées à des occasions de "socialisation au travail ", où l'on veille au respect des horaires et autres contraintes (Agulhon, 2000). Certains enseignants se rangent également à ce point de vue et renoncent à toute velléité d'y évaluer des savoir-faire.

18. Cette recherche est le plus souvent assumée par l'élève lui-même, parfois difficilement, au point que certains d'entre eux se retrouvent sans lieu de formation en entreprise.

19. Ce constat rejoint l'analyse faite dans le cadre d'une précédente étude (Beduwé et alii, 2010) qui montre que la majorité des titulaires d'un bac pro ELEEC s'insère dans des emplois «d'ouvrier qualifié des entreprises artisanales".

20. Cette remarque ne vaut pas pour tous les candidats, mais rares sont ceux qui y effectuent des tâches de maintenance. Les périodes effectuées en bureau d'étude qui permettraient de confronter les élèves au traitement de plans et autres schémas électriques ainsi qu’à l'élaboration de devis sont très rares.

21. Nous nous appuyons là sur l'observation d'exposés oraux d'environs 120 candidats au baccalauréat et près d'une trentaine au CAP, autre diplôme étudié avec la même méthodologie dans le cadre de ce travail et auxquels on pourrait ajouter la trentaine d'observations d'évaluation de PFMP (baccalauréat et CAP) non abordée dans le cadre de ce texte. L'évaluation des PFMP fera l'objet d'un texte à paraitre.

22. Et ce sur quoi ils sont jugés. Les évaluations réalisées soulignent en effet ce à quoi les enseignants accordent de l'importance.

23. Ces constats sont issus d'une recherche menée sur des périodes de formation réalisées par des élèves de Brevet de Technicien Supérieur Agricole (BTSA) en alternance. Dans cette recherche, les auteurs soulignent également le travail que les enseignants réalisent pour réduire les écarts évoqués plus haut. 


\subsection{Le concret du travail comme prétexte}

Les présentations orales de dossiers de synthèse regorgent de détails concrets sur les conditions de travail, la pénibilité ou la difficulté de certaines tâches, les pressions exercées parfois par les clients, l'application occasionnelle des normes de sécurité, le poids des contraintes imposées par une recherche de maîtrise des coûts ... et bien d'autres encore qui ont rarement la faveur du jury.

En effet, les évaluateurs ne s'intéressent pas tellement à l'expérience de travail des candidats, ni aux gestes techniques qu'ils pourraient avoir acquis. Ils perçoivent ces situations en milieu professionnel (qui ne sont d'ailleurs pas nécessairement des situations de travail) plutôt comme point de départ, voire prétexte au développement d'une question ou d'un raisonnement technique.

Les questions posées par les jurys, à la suite des exposés oraux des candidats, entretiennent donc une relation plus ou moins étroite avec les activités réalisées par les candidats au cours de leur PFMP.

Comme nous venons de le préciser, les détails les plus concrets du travail et l'expérience que les candidats en ont eue n'intéressent pas vraiment les enseignants, qui stigmatisent régulièrement les exposés trop narratifs. Cependant, certains cherchent à faire préciser les activités réalisées, leurs conditions d'exercice ou encore les difficultés rencontrées. C'est alors sur le mode de "En quoi consistait ton travail ?", "Tu l'as fait tout seul?", "Combien de temps pour faire l'installation?" que trois (seulement) des enseignants rencontrés commencent en général l'interrogation orale des candidats. Est-ce l'âge de ces enseignants ou bien leur expérience en formation d'adultes qui peut expliquer la plus grande attention qu'ils accordent aux activités réalisées par les candidats? Il est difficile d'apporter une réponse définitive à cette question. En effet, il ne faut pas s'y méprendre, car si les premières questions semblent très concrètes (et relatives au travail réalisé dans une pluralité de dimensions), celles qui suivent abordent très rapidement les attendus de l'épreuve ( $c f$. infra). En somme, faire préciser au candidat le travail qu'il a effectué n'est qu'un point de départ bien vite abandonné au profit d'une interrogation bien plus technique.

\subsection{Identifier et étudier un problème technique}

Pour une grande majorité d'enseignants, l'exercice demandé aux candidats dans le cadre de la sous-épreuve orale consiste à identifier et traiter un problème technique. Que les activités réalisées soient des activités d'exécution (voir plus haut) ne constitue en aucun cas, pour les évaluateurs, un obstacle à l'exercice. L'évaluation ne porte pas en effet sur la nature des activités réalisées, celles-ci semblent secondaires, mais sur la nature et l'intérêt « technique » du problème évoqué. Le lien avec les activités conduites 
en PFMP peut donc être plus ou moins étroit, ou bien le problème en question peut être inventé de toutes pièces si les périodes réalisées ne se prêtent pas à l'exercice :

"De toute façon, quand on fait une saignée, n’importe quoi, on va mettre un conduit à l'intérieur, ce conduit est normalisé. Il correspond à un certain nombre de caractéristiques et il peut nous parler de ça..." (enseignant de LP, 60 ans, BTS, 10 ans d'expérience professionnelle dans le privé )

"Non, mais tu dois inventer, c'est une histoire. Tu as les petits bouts de ce que tu as fait, tu inventes une histoire de ça, mais que ce soit cohérent. Une histoire technique, parce que moi, je vais te noter la partie... les expressions techniques, le vocabulaire technique." (enseignant de LP, épreuve ponctuelle ${ }^{24}$ )

Précision qui a son importance : dans ces conditions, le candidat n'est pas invité à "verbaliser " son expérience, c’est-à-dire à " réfléchir son action " (Schön, 1994). Il ne s'agit pas non plus pour lui de "prendre l'expérience pour objet " ou encore "d'expérience au carré" comme une façon de faire advenir cette expérience à la conscience afin qu'elle constitue une source de développement (Clot, 2003). Il est plutôt sommé de développer (voire de plaquer), quelle que soit l'activité déployée lors de la PFMP, un discours technique doté de règles propres. Ce discours « attendu » et normé peut cependant prendre diverses formes.

Le jury peut attendre du candidat qu'il justifie ou argumente oralement des choix d'installation (ou de composants). A l'occasion, les candidats sont invités, par un jeu de questions, à préciser les caractéristiques techniques des composants évoqués, à énoncer les lois de l'électrotechnique ou bien les normes d'installation susceptibles d'expliquer les choix accomplis.

D'autres fois, la relation avec les périodes de formation en milieu professionnel perd encore davantage de sa centralité, au profit d'une interrogation encore plus scolaire. C'est en particulier le cas lorsque les activités (ou l'étude de cas) sont insuffisamment précisées, lorsque le jury cherche à déterminer de manière rapide le " niveau " du candidat ou bien lorsque le candidat n'a pas réalisé de manière satisfaisante la présentation demandée. Les questions du jury se succèdent alors, parfois du tac-au-tac. La présentation du candidat et l'activité en PFMP sur laquelle elle s'appuie deviennent prétexte à une interrogation pouvant aller jusqu'aux questions de cours. Celle-ci peut balayer le programme en portant successivement ou alternativement sur la connaissance des langages techniques (en particulier le schéma électrique), des normes et procédures à utiliser, des fonctions ou principes de fonctionnement d'appareils ou de composants électriques :

"... mais comment il détecte? Par quel principe? Parce que là, c'est le principe de tout détecteur... mais là, c'est un détecteur particulier. Vous en connaissez d'autres types de détecteur?" (enseignant de LP, 45 ans, DUT, PLP).

24. Lors des épreuves ponctuelles, nous n'avons pu réaliser d'entretiens à caractère biographique avec les enseignants. 
"Une fois la consignation faite... le départ était donc hors tension, vous avez effectué une mise à la terre et un court-circuit. Donc, en quoi ça consiste et pourquoi on fait ça et à quel endroit on le fait?» (enseignant de LP, 40 ans, BTS puis licence, admissible au CAPET et admis PLP)

On peut aussi demander au candidat de dessiner, au tableau, un schéma unifilaire d'une partie d'installation électrique ou bien la signification d'un symbole. Ainsi, on attend du candidat qu'il se montre à la " hauteur " d'un échange technique. D'où les attentes de certains examinateurs pour que les candidats "parlent en tant que technicien", emploient les termes idoines ou précisent la définition de certains mots.

Les maitres mots de l'interrogation en épreuve orale sont aussi "principes » et "pourquoi ? ". Attend-t-on des candidats qu'ils s'extraient de la singularité et du concret des situations professionnelles pour mieux retrouver, dans les problèmes techniques évoqués, les principes généraux de l'électrotechnique, des phénomènes électriques ainsi que du fonctionnement des appareillages ? La démarche attendue semble au contraire significative des méthodes déductives propres à la transmission du savoir scientifique qui se sont diffusées à l'enseignement professionnel (Tanguy, 1991). Elle peut aussi être illustrative d'un enseignement " technologique " qui vise à transmettre les techniques comme autant de modes d'action réglés, ordonnés et applicables en toute circonstance (Cornu, 200125). Un enseignement "technologique " dont la conception rationalisante a cherché à se défaire des savoir-faire pour mieux les confiner aux «petits métiers artisanaux ». Ce même enseignement a, depuis longtemps maintenant, cherché à réintroduire ces savoir-faire sous la forme de stages ou de rapprochement avec les entreprises (Deforge, 1991), mais quelle place leur est réellement accordée ? L'examen des évaluations réalisées dans le cadre des épreuves professionnelles montre pourtant que les évaluateurs se méfient des rapports concrets, voire sensoriels, des candidats aux objets et dispositifs techniques ${ }^{26}$ et privilégient la technique aux gestes pratiques. Certes, l'enseignement, fusse-t-il professionnel, ne peut transmettre "l'intelligence pratique ". Cette dernière, "immergée dans une pratique( ...) ne (...) se soucie à aucun moment de justifier sa démarche " (Detienne et Vernant, 1978), contrairement à ce qui est attendu des candidats dans l'épreuve orale (ou le dossier). Elle est tout ce qui, en l'espèce, n'est pas valorisé par les évaluateurs : une "confrontation avec l'objet dans sa matérialité, en situation, replacé dans son contexte naturel et social. " (Cornu, 2001).

L'alternance sous statut scolaire, instaurée dans ce bac pro et pour ce que les évaluations en montrent, ne fait que renforcer les contenus transmis (le curriculum caché en quelque sorte) en établissement. Des contenus qui véhiculent une conception rationalisante de la technique, dont l'épreuve technologique « étude d'ouvrage » constitue la référence. Cette

25. Ce dernier cite Halbwachs : "une technique dit ce quill faut faire... les règles opèrent mécaniquement par leur rigidité et leur généralité ".

26. Nous faisons allusion à tous les détails concrets que livrent les candidats, signes de ce qu'ils retiennent de ces périodes de formation en entreprise mais qui n'intéressent qu’à la marge les évaluateurs. 
dernière est d'ailleurs largement évoquée ${ }^{27}$ par les enseignants au cours de l'épreuve orale (en CCF surtout), comme si elle n'était qu'une forme de préparation à l'épreuve écrite, comme d'ailleurs les épreuves pratiques (voir plus haut).

Les jugements portés sur les candidats, à l'issue de l'épreuve orale et au cours des délibérations, confortent le privilège accordé aux savoirs techniques : le futur bachelier se doit d'être technicien ; à défaut, il est très vite conduit vers un statut d'exécutant, réservé aux futurs titulaires d'un CAP :

"... c'est quand même pas très professionnel, pas très technologique... ça veut dire que c'est vraiment un exécutant. Mais un exécutant, c'est un niveau CAP, ce n'est pas un niveau bac " (enseignant de LP, 55 ans, BTS, enseignant dans le privé puis PLP interne, 2 ans d'activité en tant qu'assistant ingénieur)

Comme on le voit dans cette dernière citation, "professionnel » devient ici synonyme de " technologique ». Le technicien qu'il s'agit de former et d'évaluer est capable d'analyse et d'autonomie (définie comme capacité à opérer des choix et à les justifier ${ }^{28}$ ). Il est aussi capable de maîtriser un langage spécifique (technique), le schéma électrique par exemple, dont les dossiers de synthèse ne sauraient se passer. Les intitulés des diplômes ainsi que les descriptifs d'activité usent désormais fréquemment de ce qualificatif de technicien. Les enseignants, s'ils ne sont pas à l'origine de cette dichotomie exécutant/technicien, la reprennent volontiers et contribuent, ce faisant, à la faire exister comme une évidence.

\section{Conclusion}

Lanalyse des pratiques d'évaluation des enseignants révèle la prépondérance qu'ils accordent aux savoirs et langages techniques ainsi qu’aux démarches formalisées dans le cadre des épreuves professionnelles du baccalauréat Eleec (Electrotechnique Energie Equipements Communicants).

Cette primauté résulte de déterminations plurielles et de natures différentes. Le coefficient attribué à l'épreuve "technologique » nationale (coeff. 5, soit autant que les trois épreuves "pratiques ») signale en premier lieu l'importance que lui accordent l'administration, les corps d'inspection. De plus, l'étude de systèmes, valorisée par les enseignants, donne forme à la fois aux contenus de l'ensemble des épreuves et aux pratiques d'enseignement et d'évaluation. Ces contenus et pratiques sont également en partie déterminés par les situations (matérielles) dans lesquelles sont placés les candidats dans le cadre des épreuves pratiques notamment. Enfin, les profils des enseignants, qui se sont homogénéisés, contribuent à l'effet de ce "modèle pédagogique » fondé sur l'étude d'installations techniques. Les enseignants y adhèrent d'autant plus qu'ils sont eux-mêmes issus des filières techniques et scientifiques de l'enseignement supérieur.

27. Avec des remarques du style : «tu reverras ce point pour l'épreuve technique... ».

28. D'où les demandes formulées aux candidats visant à justifier les installations électriques qu'ils évoquent. 
Ainsi, les épreuves professionnelles apparaissent davantage comme la sanction d'un enseignement technologique que d'un enseignement professionnel de "niveau IV »; c'est-à-dire plus un enseignement centré sur l'étude de systèmes techniques qu'un enseignement en rapport avec le travail privilégiant la pratique ou l'action.

Cela souligne peut-être la permanence des représentations véhiculées dans la nomenclature des niveaux de formation et qui veut qu'au niveau IV de formation initiale soit attaché " un diplôme technique ou de technicien ". Mais cela marque aussi la persistance d'une conception rationalisante de l'enseignement qui peine à intégrer la dimension professionnelle et que l'introduction d'une alternance " sous statut scolaire »- dont la durée est certes limitée ${ }^{29}$ - a peu modifiée. Finalement, loin d'avoir " revalorisé » l'enseignement professionnel, la création du baccalauréat étudié est venue conforter des évolutions constatées avec la création du BEP (Brevet d'Enseignement Professionnel), dont l'enseignement a privilégié les savoirs techniques au détriment des savoirs professionnels (Tanguy, 1983).

La forme prise par l'enseignement, dans le baccalauréat professionnel Eleec, a été abordée comme le produit d'un ensemble de déterminations. Ces dernières semblent fournir un cadre à partir duquel pourraient être pensées les conditions d'une généralisation, à d'autres spécialités industrielles, des constats établis pour ce baccalauréat. Il resterait à construire un cadre similaire pour les enseignements des spécialités tertiaires, que l'on connait moins.

Les constats précédents peuvent aider à comprendre les poursuites d'études qui concernent de nombreuses spécialités de baccalauréats professionnels. En effet, même avant la réforme de 2009, les différents taux de poursuite d'études dont on disposait ${ }^{30}$ confirment ce phénomène. L'enseignement reçu, plus technique que professionnel, n'est-il pas de nature à préparer les bacheliers professionnels, voire à les inciter, à prolonger leur scolarité dans l'enseignement supérieur, technique en particulier ? Il conviendrait alors de relativiser le poids accordé aux stratégies familiales et individuelles dans l'allongement des scolarités. En outre, et de ce point de vue, la réforme de 2009 pourrait être envisagée comme l'inscription, dans un texte, d'une réalité.

\section{Bibliographie}

Agulhon C. (2000), «L'alternance : une notion polymorphe, des enjeux et des pratiques segmentées ", Revue française de pédagogie, $\mathrm{n}^{\circ} 131$, pp. 55-63.

Barbier J.-M. (dir.) (1996), Savoirs théoriques et savoirs d'action, Paris, PUF.

29. Rappelons que les PFMP occupent 22 semaines sur les trois années de formation (soit environ 90 semaines au total).

30. On pense notamment à ceux de la DEPP (voir la note d'information 2012/4). 
Béduwé C., Fourcade B., Legay A., Molinari M., Ourtau M., Paddeu J., Quinterro N., Sechaud F. (2010), "Les baccalauréats professionnels de l'industrie à la veille de la réforme ", Paris, CPC études $\mathrm{n}^{\circ} 2$, Tome 1.

Bidet A., Borzeix A., Pillon T., Rot G., Vatin F. (2006), Sociologie du travail et activité. Toulouse, Octarès éditions.

Canonge F., Ducel M. (1969), La pédagogie devant le progrès technique, Paris, PUF.

Chapoulie J.-M. (2000), « Le travail de terrain, l'observation des actions et des interactions, et la sociologie ", Sociétés contemporaines, $\mathrm{n}^{\circ} 40$.

Clot Y. (préf.), Vygotski Lev S. (2003), « La conscience comme liaison », in Vygotski Lev S., Conscience, inconscient, émotions, Paris, La Dispute.

Cornu R. (2001), Education, savoir et production, Bruxelles, Editions de l'Université de Bruxelles.

Darré J.-P. (1999), La production de connaissance pour l'action : arguments contre le racisme de l'intelligence, Paris, Editions de la Maison des sciences de l'Homme/ INRA.

Deforge Y. (1989), "Simondon et les questions vives de l'actualité (Postface) ", in Simondon, Du mode d'existence des objets techniques, Aubier.

Deforge Y. (1991), « Enseignements techniques, enseignements professionnels, enseignements technologiques ", in Perrin J.(dir.), Construire une science des techniques, Limonest, L'interdisciplinaire.

Delbos G., Jorion P. (1990). La transmission des savoirs, Paris, Maison des sciences de l'Homme, $2^{\circ}$ édition

Detienne M., Vernant J.-P. (1978), Les ruses de la raison. La métis des Grecs, Paris, Flammarion

Eckert H., Veneau P. (2000), « Le rapprochement de l'école et de l'entreprise dans l'enseignement technique : sur les limites d'une rationalisation volontariste ", Revue française de pédagogie, ${ }^{\circ} 131$, pp. 33-41

Figari G., Tourmen C. (2006), «La référentialisation : une façon de modéliser l'évaluation de programme, entre théorie et pratique ", Mesure et évaluation en éducation, vol. 29, $\mathrm{n}^{\circ} 3$, pp. 5-25

Formation Emploi (1995), dossier «Baccalauréat professionnel », n 49, janvier-mars, La Documentation française.

Forquin J.-C. (2008), Sociologie du curriculum, Rennes, PUR

Fourcade B. (1997), «La création des premiers baccalauréats professionnels métallurgie : maintenance, productique, électrotechnique ", in Moebus M. et Verdier E. (dir.) Les diplômes professionnels en Allemagne et en France, L'Harmattan . 
Fourcade B., Ourliac G., Ourtau M. (1992), « La négociation des diplômes technologiques : les commissions professionnelles consultatives ", Formation Emploi, n ${ }^{\circ} 39$, pp. 45-55, La Documentation française.

Pelpel P., Troger V. (1993), Histoire de l'enseignement technique, Paris, Hachette

Quenson E. (2009), “Les diplômes transversaux peinent à s’imposer sur le marché du travail ", Formation Emploi, n 106 , pp. 25-41, La Documentation française.

Quéré L. (1997), " La situation toujours négligée ? ", Réseaux, n 85, pp. 163-192

Schön D. (1994), Le praticien réflexif: à la recherche du savoir caché dans l'agir professionnel, Montréal, Les éditions Logiques.

Tanguy L. (2002), « La mise en équivalence de la formation avec l'emploi dans les IV et V plans (1962-1970) ", Revue française de sociologie, $n^{\circ}$ 4, pp. 685-709.

Tanguy L. (1991), L'enseignement professionnel en France, Paris, PUF.

Tanguy L. (1983), "Les savoirs enseignés aux futurs ouvriers », Sociologie du travail, ${ }^{\circ}$, pp. 336-354.

Veyrac H., Asloum N. (2009), " Les tâches appropriées des professeurs d'enseignement professionnel : illustration d'un hiatus entre travail en entreprise et formation ", Activités, $\mathrm{n}^{\circ}$ 1, vol. 6, pp. 69-86. 